\title{
Nova Scientia
}

\section{Revista Electrónica Nova Scientia}

\section{Talento verde y cadenas de suministro verdes: ¿existe una relación significativa? Green talent and green supply chain: is there a significant relationship?}

\section{María del Carmen Torres-Salazar ${ }^{1}$, Ana Esther Escalante- Ferrer $^{2}$, Elías Olivares-Benítez ${ }^{1}$ y Juan Carlos Pérez-García ${ }^{1}$}

${ }^{1}$ Universidad Popular Autónoma del Estado de Puebla

${ }^{2}$ Universidad Autónoma del Estado de Morelos

México 


\section{Resumen}

Introducción: En este texto se presentan resultados estadísticos de la relación entre directivos con características de talento verde y la implementación de prácticas de cadena de suministro verde. Debido a que existen investigaciones sobre la importancia de la visión de la alta dirección para la adopción de prácticas verdes; y sin embargo ninguna da cuenta de las características personales del directivo, ni tampoco de la relación significativa entre estas características y la puesta en marcha de prácticas de cadena de suministro verde, esta es la aportación de la presente investigación. El trabajo se desarrolló en empresas manufactureras con más de diez trabajadores de la zona oriente del estado de Morelos México. La caracterización del término talento verde se reportó en el artículo, Talento verde: caracterización y búsqueda, publicado en el número 14 de esta revista.

Método: El método utilizado consistió en la construcción de una encuesta dividida en tres categorías que posteriormente se convirtieron en variables: prácticas de cadena de suministro verde, presiones institucionales y talento verde. Las dos primeras se adaptaron de la encuesta de Zhu y Sarkis (2007) y la tercera surgió de la representación social de talento verde, producto de una fase de esta investigación. La encuesta se validó con un grupo de empresarios miembros de la Cámara Nacional de la Industria de la Transformación (CANACINTRA) capítulo Morelos. El instrumento validado se aplicó a 26 directivos de empresas manufactureras con más de diez trabajadores de la zona oriente de Morelos, con el fin de probar la relación entre directivos con características de talento verde y la puesta en marcha de prácticas de cadena de suministro verde. Para ello se utilizaron como herramientas estadísticas la correlación de Pearson y la regresión lineal múltiple.

Resultados: Las prácticas de cadena de suministro verde de las empresas manufactureras de la zona oriente del estado de Morelos se encuentran en etapa de planeación. Los encuestados de las empresas reconocen características de talento verde en sus directivos de mayor jerarquía. Las presiones institucionales (mercado, regulación y competidores) son importantes para las compañías que participaron en el estudio no obstante la dificultad estriba en ponerlas en marcha.

Conclusión: Talento verde y prácticas de cadena de suministro verde son variables que se correlacionan, es decir, existe una relación estadísticamente significativa entre ambas variables. A través del método de regresión lineal múltiple se afirma que las presiones institucionales: mercado, regulación y competidores, son causa de la implementación de prácticas de cadena de 
suministro verde. Estas presiones institucionales y la presencia de características de talento verde en directivos, se combinan para la implementación de prácticas de cadena de suministro verde en las empresas manufactureras con más de diez trabajadores de la zona oriente del estado de Morelos, México.

Palabras Clave: talento verde; cadenas de suministro verde; presiones institucionales; empresas manufactureras; México

Recepción: 08-11-2015 


\section{Abstract}

Introduction: In this text statistical results of the relationship between managers with features green talent and implementing practices of green supply chain are presented. Because there research on the importance of vision High direction for the adoption of green practices; yet no realizes the personal characteristics of the management, nor the significant relationship between these characteristics and implementation practices of green supply chain, this is the contribution of this research. The work was developed in manufacturing companies with more than ten workers in the eastern part of the state of Morelos Mexico. The characterization of the term green talent was reported in the article, Green Talent: characterization and research, published in issue 14 of this magazine.

Method: The method involves the construction of a survey divided into three categories conviertieron later in variables: practice green supply chain, green talent and institutional pressures. The first two were adapted survey Zhu and Sarkis (2007) and the third emerged from the social representation of green talent, due to a phase of this research. The survey was validated with a group of entrepreneurs from the National Chamber of Industry (CANACINTRA) Chapter Morelos. The validated instrument to 26 executives of manufacturing companies was applied with more than ten workers in the eastern part of Morelos, in order to test the relationship between characteristics of green managers with talent and practical implementation of green supply chain. For this were used as statistical tools Pearson correlation and multiple linear regression.

Results: Practice green supply chain of manufacturing companies in the eastern area of the state of Morelos are in the planning stage. Respondents from companies recognize green features of talent in their senior executives. Institutional pressures (market regulation and competition) are important for companies that participated in the study despite the difficulty lies in implementing them.

Conclusion: Green talent and practice green supply chain are variables that correlate, i.e., there is a statistically significant relationship between the two variables. Through multiple linear regression method states that institutional pressures: market regulation and competition, are due to the implementation of practices of green supply chain. These institutional pressures and the presence of features green talent in management, combine to implementing practices of green 
supply chain in manufacturing companies with more than ten workers in the eastern part of the state of Morelos, Mexico.

Keywords: green talent, green supply chains, institutional pressures, manufacturing companies, Mexico. 


\section{Introducción}

El factor humano, incluyendo a los altos directivos, es de suma importancia en la implementación de cualquier tipo de política empresarial. Es común entre las áreas administrativas afirmar que si la alta dirección de la organización está convencida de algún programa, los resultados de dicho programa serán exitosos, de lo contrario, pueden estar condenados al fracaso.

Siguiendo con esta lógica, el presente artículo busca cuantificar la relación entre directivos que muestran características de talento verde con la implementación de prácticas de cadena de suministro verde.

Talento verde es una expresión poco utilizada en la terminología académica; la primera fase de la investigación consistió en caracterizar este término por medio de la metodología de las representaciones sociales y la construcción de preguntas que indagaran sobre dichas características en directivos de empresas. Para la segunda fase de la investigación, se construyó una encuesta adaptando las categorías sobre prácticas de cadena de suministro verde y presiones institucionales de Zhu y Sarkis (2007) y se complementó con preguntas de talento verde que fue validada estadísticamente con un grupo de empresarios de la Cámara Nacional de la Industria de la Transformación (CANACINTRA), capítulo Morelos, México.

La encuesta validada se aplicó a un grupo de directivos de empresas de la zona oriente del estado de Morelos México, cuyos resultados nos indican que hay una relación significativa entre directivos con características de talento verde y la implementación de prácticas de cadena de suministro verde. Este es el primer trabajo de esta naturaleza que se aplica en esta zona del país.

En este reporte, la teoría institucional se presenta como andamiaje sobre las transformaciones en las políticas empresariales para explicar la adopción de prácticas verdes. Siguiendo con la parte teórica se desarrollan los conceptos y la evolución de cadenas de suministro, cadenas de suministro verde y talento verde. Posteriormente se muestran las hipótesis que guiaron la investigación. Más adelante se despliega la metodología utilizada para someter dichas hipótesis a comprobación. Ésta da cuenta de la construcción de la encuesta así como de la validación de la misma. En el apartado siguiente se describe la aplicación del instrumento. En penúltimo lugar se exponen los resultados cuantitativos y las pruebas de hipótesis, para finalizar con las conclusiones. 


\section{Teorías sobre cambios en las políticas empresariales}

Existen cuando menos tres teorías sobre las causas por las que las empresas pueden generar una transformación en sus políticas de trabajo. Barney (1991), propone la teoría de los recursos, que sostiene que las empresas más grandes, las cuales a menudo tienen mayor capital y mejores capacidades, son las que adoptan cambios debido a que son más visibles y se ven más comprometidas a satisfacer las expectativas sociales, en este caso de desempeño ambiental. Emerson (1962), presenta la teoría de la dependencia, la cual se fundamenta en la subordinación que se crea entre socios de intercambio, en la cual se ejerce cierta influencia entre los eslabones de la cadena para hacer cambios relevantes en sus formas de trabajo. La tercera es la teoría institucional propuesta por DiMaggio y Powell (1983), la cual se eligió para sostener este proyecto, debido a que incluye la teoría de la dependencia y otras causas, tales como la normatividad oficial que aplican los gobiernos y el seguimiento de las políticas de sus competidores. Tanto la dependencia como la legislación y los competidores son causas para implementar un cambio en las empresas, en este caso, la adopción de prácticas amigables con el medio ambiente. Por lo anterior y sus alcances se considera que esta teoría es la más completa, ya que contempla tres principios de transformación que se pueden aplicar al enverdecimiento empresarial.

De acuerdo con DiMaggio y Powell (1983), los cambios en las empresas, en este caso la alineación ambiental, puede verse influida por tres presiones: normativas, coercitivas y miméticas. Las presiones normativas son aquellas que imponen los requisitos del cliente y se asocian con la profesionalización de los procesos. El gran comprador (empresas focales como las cadenas de supermercados), aprovecha la ventaja del prestigio de su firma e impone a sus proveedores una serie de requisitos que deben cumplir para ostentarse como cadenas productivas que cuidan el planeta. Dentro de estos requisitos puede figurar, entre otros, la certificación ISO 14000. Las presiones coercitivas, que ejercen influencia política y buscan legitimidad, tienen que ver con las leyes que imponen los gobiernos para el cumplimiento de normas medio ambientales. En México, la miscelánea fiscal 2015 incluye un “impuesto verde”, que cobrará aproximadamente \$3.2 USD (Centro de Estudios en Finanzas Publicas 2015), por tonelada de bióxido de carbono emitida, impuesto que forma parte de la estrategia de cambio climático que compromete al país a reducir un 30\% la emisión de gases de efecto invernadero para el año 2020 
(SEMARNAT 2013). Así también, las presiones miméticas, que son la respuesta estándar a la incertidumbre por medio de la innovación, son aquellas que adoptan las empresas como imitación a sus competidores más exitosos. La adopción de prácticas verdes puede generar a largo plazo un aumento en las utilidades de las empresas (Zhu y Geng 2001). La emulación de los competidores puede deberse al espejismo de ese aumento en las utilidades y generalmente se realiza a través del benchmarking. Por otra parte, existen otros factores para adoptar la alineación ambiental en las empresas, entre ellos se encuentran el liderazgo y el compromiso de la alta dirección, los cuales se enfatizan en este trabajo a través del término talento verde.

\section{Cadenas de suministro}

Una cadena de suministro es "una combinación de procesos, funciones, actividades, relaciones y vías por las que los productos, los servicios, la información y las transacciones financieras se desplazan dentro de y entre las empresas a partir del fabricante original hasta el usuario o consumidor final” (Gattorna 2006, 40). También se dice que: “una cadena de suministro está formada por todas aquellas partes involucradas de manera directa o indirecta en la satisfacción de una solicitud de un cliente. Incluye no solamente al fabricante y al proveedor, sino también a los transportistas, almacenistas, vendedores al detalle (o menudeo) e incluso a los mismos clientes” (Chopra y Meindl 2008, 3). En resumen, una cadena de suministro es un grupo de empresas que interactúan dentro y entre sí con el objetivo de satisfacer a un cliente a través de un producto o un servicio. El grupo de empresas envuelve a proveedores tradicionales, proveedores financieros y de logística, clientes y por supuesto a la organización principal, es decir, la empresa focal. La figura 1 nos muestra la configuración de una cadena de suministro.

Figura 1. Cadena de suministro última

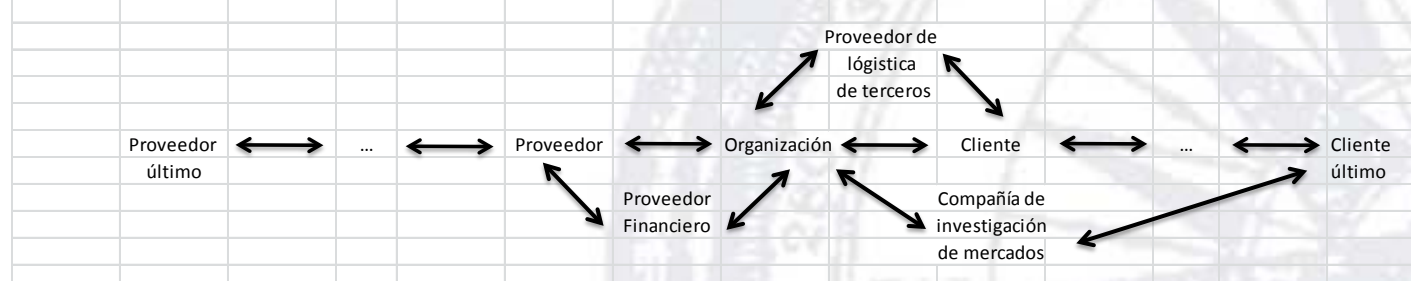

Fuente: Murphy y Knemeyer $(2015,77)$ 
La interacción entre empresas comprende diversos procesos, dentro de la misma empresa y entre las empresas. A la integración de estos procesos se le llama administración de la cadena de suministro. Las definiciones de administración de la cadena de suministro reportadas en la literatura (Ahi y Searcy 2013), exhiben las siguientes características: enfoque de flujo, enfoque de coordinación, enfoque de partes interesadas, enfoque de relaciones, enfoque de eficiencia y enfoque de rendimiento, es decir, la administración de una cadena de suministro comprende el flujo de materiales, servicios e información coordinado a través de las empresas que forman la cadena y dentro de cada empresa participante, que incluye a proveedores y clientes, los cuales se relacionan para la creación de valor de forma eficiente y que permite alcanzar los objetivos planteados.

Las cadenas de suministro se han transformado a través del tiempo, incluyendo factores diversos. Una de sus transformaciones más trascendentes es la adopción de prácticas medio ambientales en sus procesos, lo que se ha denominado cadenas de suministro verdes.

\section{Cadenas de suministro verdes}

La respuesta de las cadenas productivas a la problemática medio ambiental es el enverdecimiento de sus procesos. Esta transformación se engloba en el término cadena de suministro verde. Este término se empezó a utilizar a finales de los años 90. Handfiel (1997), dice que una cadena de suministro verde es la aplicación de los principios de gestión ambiental en todo el conjunto de actividades a través de todo el ciclo de pedido del cliente, incluyendo diseño, aprovisionamiento, fabricación y montaje, embalaje, logística y distribución. Beamon (1999), explica que la estructura tradicional de la cadena de suministro debe ampliarse para incluir mecanismos para la recuperación del producto, esto es, considera a la logística inversa. Hoeck (1999), manifiesta que la logística inversa puede no ser suficiente y que un enfoque en toda la cadena de suministro es más relevante para comprender el impacto de las prácticas empresariales en el medio ambiente. Diversos autores hacen otras aportaciones a la definición de cadena de suministro verde desde diferentes puntos de vista, por ejemplo, Lakhal et al. (2007) dice que una cadena de suministro verde puede ser caracterizada por los cinco círculos de los Juegos Olímpicos: cero emisiones, cero residuos en las actividades, cero residuos de los recursos, cero usos de sustancias tóxicas, cero residuos en el ciclo de vida del producto, además de entradas y salidas verdes. Sin embargo, la definición más citada es la de Srivastava (2007) que habla de la unificación de prácticas medio 
ambientales a lo largo de los procesos (diseño, proveeduría, fabricación, entregas al cliente final), y por tanto de los integrantes de la cadena, considerando además un cierre, que es la disposición final del producto y que permite reconocer a una cadena de suministro verde como un proceso de ciclo cerrado, donde teóricamente se devuelve el producto al final de su vida útil a los procesos de fabricación donde se reprocesa: algunos componentes se reciclan, otros se re-manufacturan, otros se re-usan, y algunos, se espera que sean los menos, se desperdician.

El catálogo de prácticas verdes que se realizan en las cadenas de suministro, es tan extenso como las definiciones de cadenas de suministro verdes existentes e incluyen, de acuerdo con Hervani et al. (2005, 334), Compra Verde + Fabricación Verde / Gestión de Materiales + Distribución Verde / Mercadotecnia + Logística Inversa. El Saadany et al. (2011, 1203) contempla la reducción de energía, del uso de material bruto y de la generación de residuos, y el aumento de las opciones de recuperación del producto. Sin embargo, las prácticas que proponen Zhu y Sarkis (2007) son las que más se han estudiado empíricamente, por Laosirihongthong, Adebanjo y Choon Tan (2013); Ben Brick, Mellah y Belaid (2013) y Lee (2008). Dichas prácticas son: de administración interna (incluye el compromiso de los directivos y las certificaciones), de compra verde (cooperación con los proveedores), de eco-diseño (diseño de productos con consumos reducidos de material y energía, reutilización y reciclaje, entre otros), de cooperación con los clientes (para el eco-diseño, la producción más limpia y el empaque verde) y de recuperación de la inversión (venta de exceso de inventario, chatarra y maquinaria en desuso). En la figura 2 se muestran estas prácticas verdes de forma desglosada. Dicha figura es resultado de la información de Zhu y Sarkis (2007). Sin embargo, en la encuesta se adicionó un bloque sobre prácticas de reducción en el nivel operacional. 
Figura 2. Prácticas de cadena de suministro verde

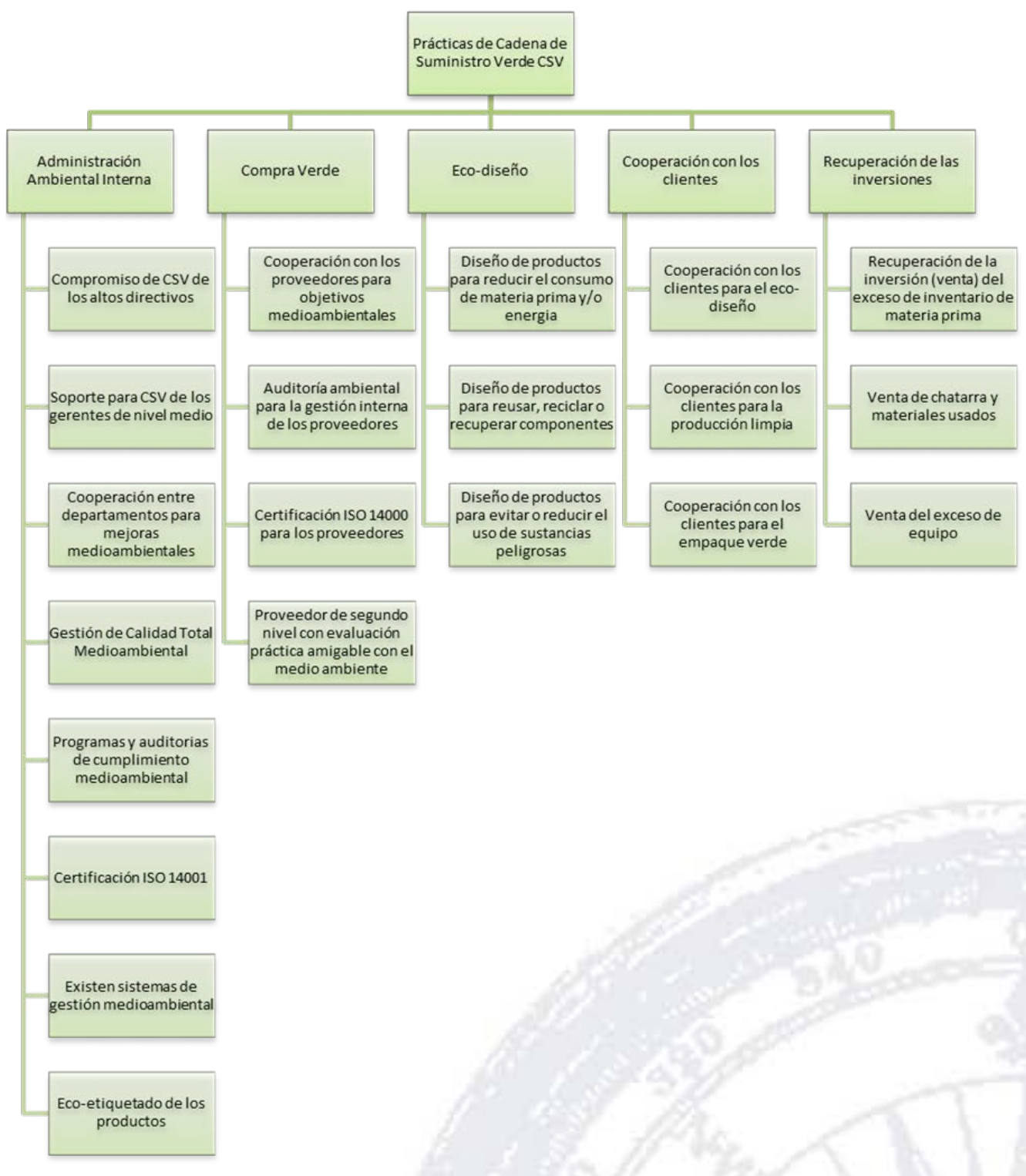

Fuente: Elaboración propia con la información de Zhu y Sarkis (2007).

\section{Talento verde}

En una fase previa de esta investigación, la cual forma parte del proyecto general, se caracterizó a partir de las representaciones sociales, el término talento verde. El resultado se muestra a continuación: 
El talento verde lo constituyen aquellas personas que tienen un compromiso con el medio ambiente que demuestran con su forma de vida. Son capaces de integrar conocimientos de diferentes áreas con el objetivo de preservar la naturaleza. Saben que el cuidado del entorno es un deber moral (responsabilidad), que lo compromete y obliga. Está consciente que con su pensar y actuar en favor del medio puede transformar a los demás (cambio cultural). Ve al mundo como un proceso complejo donde intervienen factores humanos, sociales y técnicos, lo cual ayuda a comprender nuestro hábitat. Convive o ha convivido con la naturaleza y le da un gran valor, es decir, reconoce la riqueza, la pureza y la armonía del entorno. Tiene además una gran capacidad de observación y conocimiento de los procesos ambientales (transformaciones químicas, físicas o biológicas en cualquier componente del ambiente como aire, agua o suelo) (Torres-Salazar, et al. 2015, 706).

Esta representación social de talento verde es producto de un trabajo analítico de las opiniones de un grupo de investigadores, expertos en cuestiones ambientales. La relación con los directivos de empresas está justificada por las políticas públicas nacionales de vinculación universidadempresa (CONACYT 2015). La definición anterior engloba nueve características: compromiso, integración del conocimiento, responsabilidad, cambio cultural, visión integral del mundo, convivencia con la naturaleza, valoración, capacidad de observación y procesos ambientales. Con dichas características se complementó la encuesta de la que da cuenta esta investigación y sobre la cual se trabajó estadísticamente para probar las hipótesis planteadas en la siguiente sección.

\section{Hipótesis}

El apoyo de la alta jerarquía es necesario para el éxito de cualquier programa dentro de las organizaciones. Los ejemplos más populares son los sistemas ISO de gestión de calidad y gestión ambiental, mismos que contienen un apartado que subraya el compromiso de la alta dirección para el éxito en la implementación de dichos sistemas. Diversos autores han analizado la importancia del factor humano en la implementación de políticas medio ambientales en las empresas: Daily y Huang (2001), exploran la importancia del compromiso de la alta dirección para la implementación del sistema de gestión de calidad ISO 14000; Pagell y Gobeli (2009), hablan de actitudes hacia la sostenibilidad, pero se refieren mayormente a lo que los empleados hacen dentro de las empresas; Sarkis, Gonzalez-Torre y Adenso-Diaz (2010), consideran al entrenamiento (capacitación), como efecto mediador para la adopción de prácticas medio 
ambientales; Kumar, Teichman y Timpernagel (2012), mencionan que dentro de los conductores internos para la ejecución de prácticas de cadena de suministro verde se encuentra principalmente una visión positiva de la alta dirección. Sin embargo ninguno estudia cuales son las características de estos altos directivos ni demuestra una relación estadística entre estas características y la implementación de prácticas verdes en las cadenas de suministro. Esta es la aportación de la investigación que se reporta en este artículo.

Considerando lo anterior, la pregunta que conduce este trabajo es: ¿Existe una relación significativa entre directivos que cuentan con características de talento verde y la adopción de prácticas de cadena de suministro verde? Esta pregunta generó tres hipótesis, las cuales guiaron la investigación:

H1 La presencia de características de talento verde en los directivos, se encuentra vinculada con la implementación de prácticas de cadena de suministro verde en empresas manufactureras con más de diez trabajadores de la zona oriente del estado de Morelos, México.

Estudios anteriores, acordes con la teoría institucional (Zhu y Sarkis 2007 y Laosirihongthong, Adebanjo y Choon Tan 2013), apuntan que las prácticas de cadena de suministro verde se relacionan con circunstancias normativas, coercitivas y miméticas. La segunda hipótesis se relaciona con lo anterior:

H2 Las presiones institucionales (mercado, legislación y competidores) están relacionadas de manera significativa con la implementación de prácticas de cadena de suministro verde en las empresas manufactureras con más de diez trabajadores de la zona oriente del estado de Morelos, México.

Por último, considerando que tanto las características de talento verde en los directivos como las presiones institucionales pueden ser causa de la implementación de prácticas de cadena de suministro verde, la tercera hipótesis se plantea de la siguiente manera:

H3 Las presiones institucionales y la presencia de directivos con talento verde tienen una influencia significativa en la implementación de prácticas de cadena de suministro verde en las empresas manufactureras con más de diez trabajadores en la zona oriente del estado de Morelos, México. 
La metodología empleada para someter a comprobación las hipótesis anteriores se describe a continuación.

\section{Método}

Para comprobar las hipótesis, el diseño de la investigación consideró la aplicación de una encuesta y su correspondiente análisis estadístico. De acuerdo con Kotzab (2005), la encuesta es un instrumento ampliamente utilizado en la investigación de negocios y logística. La encuesta que se utilizó en este trabajo consta de tres categorías: prácticas de cadena de suministro verde, presiones institucionales y talento verde. Tanto la categoría de prácticas de cadena de suministro verde como la de presiones institucionales fueron desarrolladas en la encuesta elaborada por Zhu y Sarkis (2007). La categoría de talento verde, de la cual no hay un antecedente, se diseñó exprofeso para esta investigación. Primeramente se procedió a caracterizar el talento verde para así obtener las preguntas que se integraron a la encuesta sobre prácticas de cadena de suministro verde y presiones institucionales. Los pasos que se siguieron y los resultados se reportaron en un trabajo previo (Torres-Salazar, et al. 2015). Las preguntas que se elaboraron a partir de la representación de talento verde y su escala de medición se muestran en la tabla 1. 
Tabla 1. Lista de preguntas sobre talento verde y su escala de medición

\begin{tabular}{ll}
\hline \multicolumn{1}{c}{ Característica } & \multicolumn{1}{c}{ Pregunta } \\
\hline Compromiso & $\begin{array}{l}\text { 1. El Director General demuestra su compromiso medioambiental en } \\
\text { su forma de actuar, por ejemplo, reciclando el papel o apagando la } \\
\text { luz cuando sale de su oficina. }\end{array}$ \\
2. El Director General utiliza los conocimientos de diversas áreas e \\
Integración del conocimiento & $\begin{array}{l}\text { incluso solicita la opinión de personal de diferentes profesiones para } \\
\text { resolver la problemática medioambiental. }\end{array}$ \\
Responsabilidad & $\begin{array}{l}\text { 3. El pensamiento del Director General considera que el cuidado del } \\
\text { medio ambiente es un deber moral. }\end{array}$ \\
Cambio cultural & 4. El Director General considera que además de una política general, \\
son primordiales las acciones individuales para cambiar el entorno.
\end{tabular}

Fuente: Elaboración propia con base en la representación social

En la segunda parte de la encuesta, se pretende indagar si existen prácticas de cadena de suministro verde. Esta parte es una adaptación de las encuestas que han aplicado varios autores en los Emiratos Árabes Unidos, Corea del Sur, China y Tailandia (Ben, Mellah y Rettab 2013; Laosirihongthong, Adebanjo y Choon 2013; Su-Yol 2008 y Zhu y Sarkis 2007). Los bloques en que se divide esta categoría son: administración ambiental interna, compra verde, eco diseño, cooperación con los clientes, recuperación de la inversión y prácticas de reducción en el nivel operacional. Las preguntas y la escala en que se midió cada bloque se muestran en la tabla 2. Es de hacer notar que el último bloque se adicionó a la encuesta original utilizada por Zhu y Sarkis (2007). En dicho bloque se pregunta sobre las prácticas de reducción en el nivel operacional que cerrarían el ciclo de la cadena de suministro. 
Tabla 2. Lista de preguntas sobre prácticas de cadena de suministro verdes y su escala de medición

\begin{tabular}{|c|c|}
\hline Bloque & Prácticas de cadena de suministro verde \\
\hline $\begin{array}{l}\text { Administración ambiental } \\
\text { interna }\end{array}$ & $\begin{array}{l}\text { 1. Respecto a la implementación y el seguimiento de prácticas } \\
\text { medioambientales dentro de la empresa, la alta dirección } \\
\text { 2. Los mandos medios (directivos de nivel operativo) apoyan la } \\
\text { implementación y el seguimiento de prácticas medioambientales } \\
\text { en la empresa } \\
\text { 3. Existe cooperación entre los departamentos de la empresa } \\
\text { para hacer mejoras medioambientales } \\
\text { 4. Existe un departamento de ingeniería ambiental, de seguridad y } \\
\text { medio ambiente o similar } \\
\text { 5. Hay programas de calidad total medioambiental en la empresa } \\
\text { 6. Hay auditorías de cumplimiento medioambiental dentro de la } \\
\text { empresa } \\
\text { 7. La empresa tiene certificación ISO } 14000 \\
\text { 8. Existe un sistema de gestión medioambiental (diferente al ISO } \\
\text { 14000) } \\
\text { 9. En la etiqueta del producto se presentan datos sobre } \\
\text { cuestiones amigables con el medioambiente (eco etiguetado) }\end{array}$ \\
\hline Compra verde & $\begin{array}{l}\text { 1. Existe cooperación con los proveedores para cumplir } \\
\text { objetivos medioambientales conjuntos } \\
\text { 2. Se hacen auditorías medioambientales a los proveedores } \\
\text { 3. Se pide certificación ISO } 14000 \text { a los proveedores } \\
\text { 4. Se hace una evaluación medioambiental a los proveedores de } \\
\text { segundo nivel (proveedores de los proveedores) }\end{array}$ \\
\hline Ecodiseño & $\begin{array}{l}\text { 1. En el diseño de los productos, se considera la reducción de } \\
\text { materiales y/o energía } \\
2 \text {. En el diseño de los productos, se considera el re-uso, el } \\
\text { reciclaje o la recuperación de componentes } \\
\text { 3. En el diseño de los productos, se considera evitar o reducir el } \\
\text { uso de sustancias peligrosas }\end{array}$ \\
\hline Cooperación con los clientes & $\begin{array}{l}\text { 1. Existe cooperación con los clientes para diseñar productos } \\
\text { amigables con el medio ambiente } \\
\text { 2. Existe cooperación con los clientes para la utilización de } \\
\text { procesos productivos amigables con el medio ambiente } \\
\text { 3. Existe cooperación con los clientes para hacer el empaque } \\
\text { menos dañino para el medio ambiente (incluye reciclaje de } \\
\text { tarimas y/o empaques múltiples) }\end{array}$ \\
\hline Recuperación de la inversión & $\begin{array}{l}\text { 1. La empresa vende el exceso de materia prima } \\
\text { 2. La empresa vende materiales usados } \\
\text { 3. La empresa vende equipo obsoleto o en desuso (chatarra) }\end{array}$ \\
\hline $\begin{array}{l}\text { Prácticas de reducción en el } \\
\text { nivel operacional }\end{array}$ & $\begin{array}{l}\text { 1. Existe algún programa de reducción de materiales y/o energía } \\
\text { en los procesos productivos } \\
\text { 2. Se recuperan materiales o componentes en algún proceso o } \\
\text { incluso en productos al fin de su vida útil } \\
\text { 3. Se utilizan materiales reciclados en alguna parte del proceso }\end{array}$ \\
\hline = Se está llevando a ca & тателье \\
\hline
\end{tabular}

Fuente: Elaboración propia con datos de Zhu y Sarkis (2007)

La tercera parte de la encuesta está centrada en las presiones por las cuales una empresa puede adoptar prácticas de cadena de suministro verde. Esta categoría también fue utilizada en la encuesta realizada por Zhu y Sarkis (2007). Los bloques, las preguntas correspondientes y su escala de medición se muestran en la tabla 3. 
Tabla 3. Lista de preguntas sobre presiones institucionales y su escala de medición

\begin{tabular}{|c|c|}
\hline Bloque & Presiones institucionales \\
\hline \multirow[t]{4}{*}{ Mercado } & 1. Exportación de sus productos \\
\hline & 2. Venta a clientes transnacionales (aun cuando \\
\hline & los productos no salgan del país) \\
\hline & 3. Políticas medioambientales de los clientes \\
\hline \multirow[t]{4}{*}{ Legislación } & $\begin{array}{l}\text { 1. Leyes internacionales de cuidado al medio } \\
\text { ambiente }\end{array}$ \\
\hline & $\begin{array}{l}\text { 2. Legislación nacional medioambiental (Ley del } \\
\text { cambio climático, impuesto verde) }\end{array}$ \\
\hline & 3. Legislación estatal medioambiental \\
\hline & 4. Legislación municipal medioambiental \\
\hline \multirow[t]{2}{*}{ Competencia } & 1. Estrategias ecológicas de los competidores \\
\hline & $\begin{array}{l}\text { 2. Actividades de cuidado al medio ambiente que } \\
\text { promueven los grupos industriales a los que } \\
\text { pertenece }\end{array}$ \\
\hline \multicolumn{2}{|c|}{ Valores de la escala } \\
\hline \multicolumn{2}{|c|}{$1=$ Nada importante } \\
\hline \multicolumn{2}{|c|}{$2=$ Poco importante } \\
\hline \multicolumn{2}{|c|}{$3=$ No se piensa en ello } \\
\hline \multicolumn{2}{|c|}{$4=$ Importante } \\
\hline \multicolumn{2}{|c|}{$5=$ Extremadamente importante } \\
\hline
\end{tabular}

Fuente: Elaboración propia con datos de Zhu y Sarkis (2007)

La versión adaptada de la encuesta para el pilotaje contenía 43 preguntas. La adaptación consistió en la traducción del inglés al español de forma que la comprendieran los ejecutivos de empresa mexicanos. Se adicionaron preguntas al bloque de administración ambiental interna y el bloque completo de prácticas de reducción en el nivel operacional. También se añadieron preguntas al bloque de legislación para considerar reglamentación internacional, nacional, estatal y municipal, las tres últimas son los niveles de gobierno que contempla el Estado mexicano. En la siguiente sección se explica la forma en que se validó el instrumento.

\section{Validación de la encuesta}

De acuerdo con Hernández-Sampieri, Fernández-Collado y Baptista-Lucio (2010), los instrumentos de recolección de datos deben reunir tres requisitos esenciales: confiabilidad, validez y objetividad. La confiabilidad, es el grado en que un instrumento produce resultados 
consistentes y coherentes. La validez, es el grado en que un instrumento en verdad mide la variable que se busca medir y la objetividad se refiere al grado en que el instrumento es permeable a la influencia de los sesgos y tendencias de los investigadores que lo administran, califican e interpretan.

Para llevar a cabo la validación de este instrumento, la encuesta se aplicó a los asistentes a la junta mensual de CANACINTRA (Cámara Nacional de la Industria de la Transformación) Capítulo Morelos, México, el día 25 de noviembre de 2014. Se obtuvieron 25 encuestas resueltas. La confiabilidad (consistencia interna), fue medida a través del Alfa de Cronbach dado que el instrumento solo se aplicó una vez. Las respuestas fueron dadas en escala Likert con variables ordinales. El análisis se realizó por cada categoría, bloque y de manera general. Todos los valores del Alfa de Cronbach fueron mayores a 0.5.

La validez se midió a través de un análisis factorial confirmatorio, que de acuerdo con PérezLópez (2008), obliga a comprobar si las variables se ajustan a un cierto modelo de forma parcial o absoluta. Para este análisis, se utilizó el método de componentes principales iteradas o ejes principales para obtener los factores. Se realizó un contraste previo para analizar la pertinencia de la aplicación del análisis factorial a través del KMO (Kaiser, Meyer y Olkin de adecuación muestral global al modelo factorial). Si el $\mathrm{KMO}<0.5$ entonces es inadecuado aplicar un modelo de análisis factorial. Solo el bloque de prácticas de reducción a nivel operacional no cumplió con este requisito y por tanto se eliminó del instrumento. Se utilizó también el Método Varimax de rotación, para obtener, a partir de la solución inicial, unos factores que sean fácilmente interpretables. En el análisis factorial se sustituye el conjunto de variables originales por los factores obtenidos con el método Varimax y como no se obtienen puntuaciones exactas para los factores, se aplica el método de máxima verosimilitud (estimación Barlett), asumiendo que los factores tienen una distribución normal con media y matriz de covarianzas dadas. La matriz de factores rotados mostró tres factores en el bloque de administración ambiental interna y dos en el bloque de talento verde, pero dado que no se encontraron valores negativos y las preguntas planteadas para cada bloque constituyen un solo constructo, no es necesario eliminar preguntas, ya que cada una cuestiona cosas diferentes. El instrumento es entonces válido y confiable. El resumen de estos análisis se muestra en la tabla 4. 
Tabla 4. Resumen del análisis para comprobar confiabilidad y validez de la encuesta

\begin{tabular}{|c|c|c|c|c|c|c|c|c|}
\hline Categoría & Bloque & No de Preguntas & Alfa de Cronbach & KMO & PV & Factores & $\begin{array}{l}\text { Matriz de } \\
\text { Factores } \\
\text { Rotados }\end{array}$ & Observaciones \\
\hline \multirow{6}{*}{$\begin{array}{c}\text { Prácticas de } \\
\text { cadena de } \\
\text { suministro } \\
\text { verde }\end{array}$} & $\begin{array}{l}\text { Administración } \\
\text { ambiental interna }\end{array}$ & 9 & 0.811 & 0.596 & 0.000 & 3 & No negativos & \\
\hline & Compra verde & 4 & 0.934 & 0.826 & 0.000 & 1 & & \\
\hline & Ecodiseño & 3 & 0.736 & 0.592 & 0.001 & 1 & & \\
\hline & $\begin{array}{l}\text { Cooperación con } \\
\text { los clientes }\end{array}$ & 3 & 0.843 & 0.673 & 0.000 & 1 & & \\
\hline & $\begin{array}{l}\text { Recuperación de } \\
\text { la inversión }\end{array}$ & 3 & 0.864 & 0.692 & 0.000 & 1 & & \\
\hline & $\begin{array}{l}\text { Practicas de } \\
\text { reducción en el } \\
\text { nivel operacional }\end{array}$ & 3 & 0.665 & 0.425 & 0.000 & & & $\begin{array}{l}\text { No se logró } \\
\text { obtener ningún } \\
\text { factor }\end{array}$ \\
\hline \multirow{5}{*}{$\begin{array}{c}\text { Presiones } \\
\text { institucionales }\end{array}$} & & 25 & 0.830 & & & & & $\begin{array}{l}\text { No es matriz } \\
\text { positiva }\end{array}$ \\
\hline & Mercado & 3 & 0.956 & 0.764 & 0.000 & 1 & & \\
\hline & Regulación & 4 & 0.969 & 0.801 & 0.000 & 1 & & \\
\hline & Competencia & 2 & 0.919 & 0.500 & 0.000 & 1 & & \\
\hline & & 9 & 0.974 & 0.848 & 0.000 & 1 & & \\
\hline Talento verde & Talento verde & 9 & 0.948 & 0.849 & 0.000 & 2 & No negativos & \\
\hline TOTAL & & 43 & 0.887 & & & & & $\begin{array}{l}\text { No es matriz } \\
\text { positiva }\end{array}$ \\
\hline
\end{tabular}

Fuente: Elaboración propia.

\section{Aplicación de la encuesta}

La aplicación de la encuesta sobre prácticas de cadena de suministro verde, se hizo considerando las empresas que se encuentran en el Directorio Estadístico Nacional de Unidades Económicas (DENUE) (INEGI 2014), y que cumplen con los requisitos de ser industrias manufactureras, con más de 10 trabajadores en el estado de Morelos, México. No se consideran las micro empresas ya que de acuerdo a la estratificación oficial para México (DOF 2009), son aquellas de cero a diez trabajadores y que por lo general, integran cadenas de suministro muy cortas. La cantidad de empresas del estado de Morelos que tienen las características de ser manufactureras y con más de diez trabajadores, encontradas en el directorio mencionado fueron 329.

Debido a que desde el año 2013, el Gobierno del Estado de Morelos tiene especial interés por detonar la zona metropolitana de Cuautla, dada su ubicación estratégica ya que colinda con el estado de Puebla y se encuentra cerca de la ciudad de México, y a que en ese año, se constituyó el proyecto Desarrollo Industrial Verde (DIVE) para desplegar en uno de los municipios de esa región (Yecapixtla), el cual tiene como objetivo aglutinar a unas 25 empresas de la rama de alimentos, bebidas, autopartes y textil. Dicho desarrollo se construye de forma sustentable y las 
empresas que se establezcan en él deberán, por lo menos, de contar con programas de ahorro de energía y la utilización de materiales no tóxicos (Gobierno del Estado de Morelos 2013). Este proyecto despertó el interés de los autores de este artículo para realizar la investigación en esta región por lo que este estudio se realizó en las empresas manufactureras de la zona metropolitana de Cuautla ${ }^{12}$.

Con el objeto de obtener una mayor tasa de respuesta, se programó una reunión con representantes de las empresas a encuestar, en donde se les explicó el objetivo de la encuesta y se les pidió que contestaran el instrumento. Dicha reunión fue co-convocada por la Escuela de Estudios Superiores de Xalostoc, perteneciente a la Universidad Autónoma del Estado de Morelos en el mes de abril de 2015. Se hizo una actualización del directorio vía telefónica, a partir de la cual se imprimieron las invitaciones personalizadas para el representante de cada empresa, pidiendo que asistiera la persona responsable de lo relativo al cuidado del medio ambiente. Dos semanas antes del evento se entregaron dichas invitaciones personalmente. A la par se mandaron correos electrónicos conservando el formato de la invitación. Dos días antes del evento, se confirmó la asistencia vía telefónica. El día de la sesión de aplicación de la encuesta, se presentaron 26 representantes de empresas, lo cual constituye 30.23\% del total de las empresas que conforman el censo publicado por el INEGI para esta zona. De acuerdo con Cristmann (2000), Melnyc, Sroufe y Calentone (2003) y Delmas y Keller (2005), para este tipo de trabajos la tasa de respuesta observada es de alrededor del $13 \%$.

Para esta aplicación, la confiabilidad fue medida nuevamente a través del Alfa de Cronbach. Se realizaron análisis por cada categoría y bloque, así como uno general. Los resultados muestran que todos los valores son mayores a 0.5 , lo cual nos indica que el instrumento es confiable. Se volvió a realizar un análisis factorial y de acuerdo con los resultados, se eliminaron las preguntas sobre legislación nacional y municipal, por lo cual los análisis estadísticos se realizaron sobre 38 preguntas. A continuación se presentan los resultados obtenidos.

\footnotetext{
${ }^{1}$ Que comprende 14 municipios y dos parques industriales. El total de empresas que cumplen con las características buscadas fue de 86 .

${ }^{2}$ La clasificación de las zonas metropolitanas Cuernavaca, Cuautla y Sur del estado de Morelos se retoma de TapiaUribe (2006).
} 


\section{Resultados}

De acuerdo con las encuestas contestadas, las empresas se dedican a diversas actividades: desde la confección de blancos hasta la elaboración de artesanías, pasando por la fabricación de partes eléctricas automotrices y la matanza de ganado, como se observa en la tabla 5. Las empresas encuestadas tienen personal ocupado desde 11 hasta más de 250 trabajadores, como se muestra en la Gráfica 1. Los datos presentan un amplio abanico de actividades, así como de personal ocupado, datos que permiten considerarlas como representativas de las firmas de la zona oriente del estado de Morelos.

Tabla 5. Clases de actividad que desarrollan las empresas encuestadas

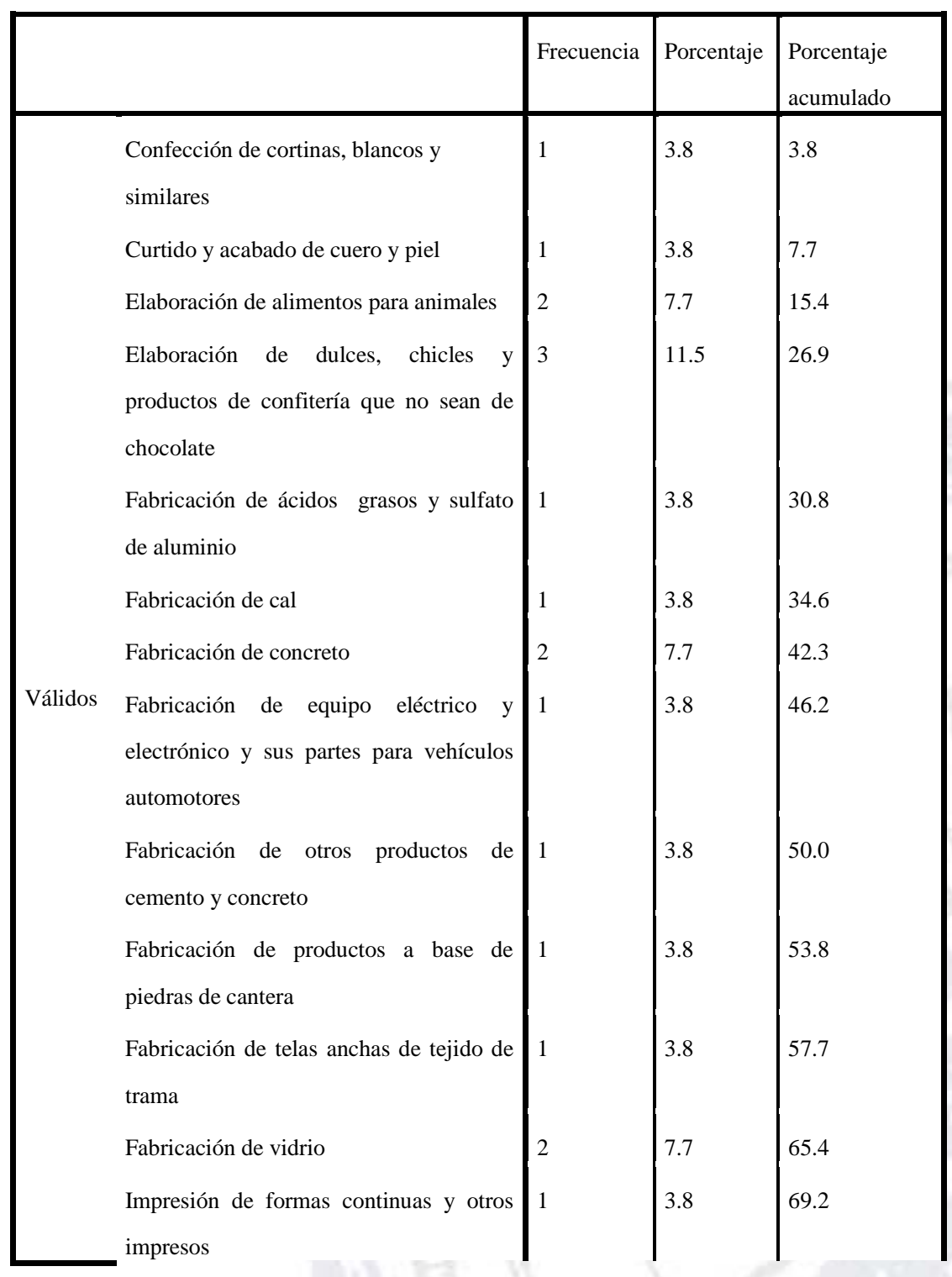




\begin{tabular}{|l|l|l|l|l|}
\hline $\begin{array}{l}\text { Matanza de ganado, aves y otros } \\
\text { animales comestibles }\end{array}$ & 2 & 7.7 & 76.9 \\
Otras industrias manufactureras & 3 & 11.5 & 88.5 \\
(artesanías) & 2 & 7.7 & 96.2 \\
Panificación tradicional & 1 & 3.8 & 100.0 \\
Purificación y embotellado de agua & 26 & 100.0 & \\
\hline
\end{tabular}

Fuente: Elaboración propia

Gráfica 1. Personal ocupado de las empresas encuestadas

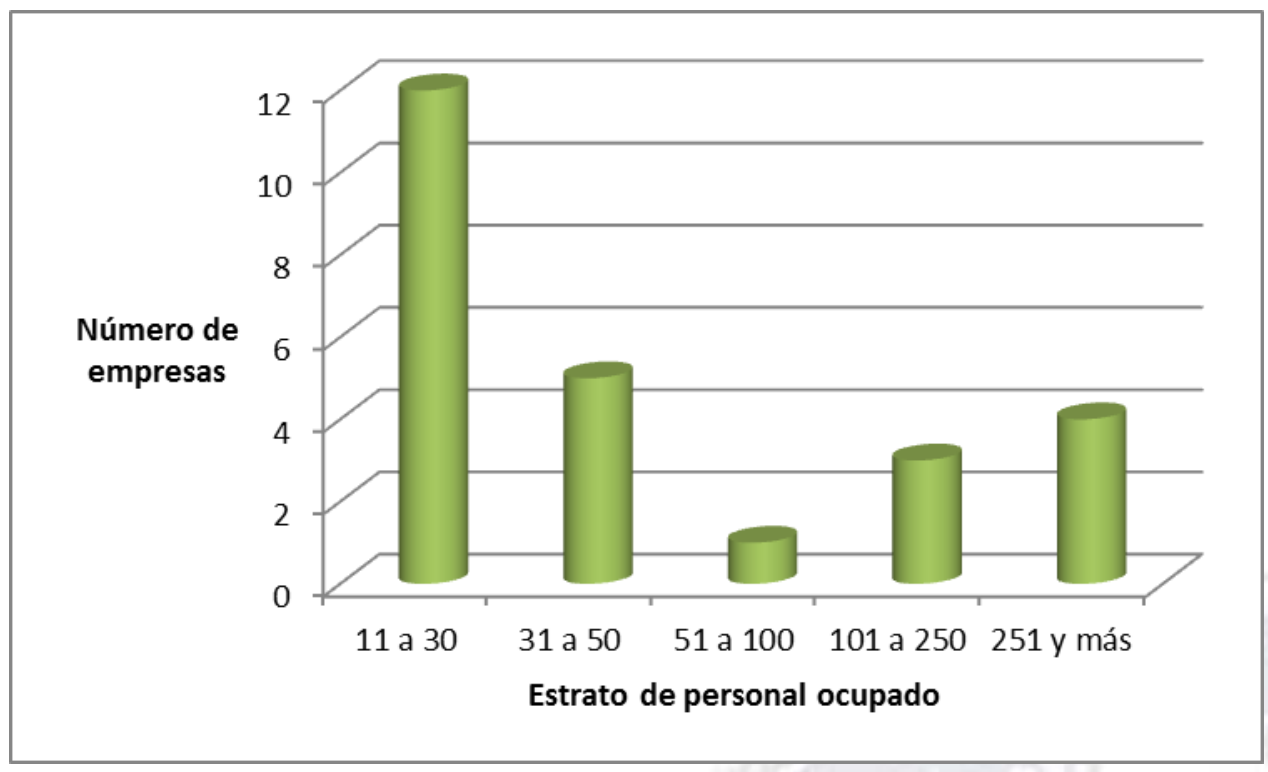

Fuente: Elaboración propia

\section{Talento verde en los directivos de las empresas encuestadas}

De acuerdo con los datos de las encuestas, los directivos de las empresas presentan características de talento verde. La media para la variable “Talento Verde” es de 3.8 (ver tabla 1 y 6), es decir, llevan a cabo en algún grado acciones que ponen en evidencia las características que definen el talento verde. De las nueve características que conforman el talento verde, la que tiene la media más alta es la responsabilidad (4.15), que considera al cuidado del medio ambiente como un deber moral. Dicha característica aunada cobra mayor sentido a las prácticas verdes si se combina con otras dos características que obtuvieron medias superiores a 4: la visión integral del mundo y el compromiso. La que obtiene la media más baja (2.96), es la característica que corresponde a procesos ambientales en la pregunta de capacitación en áreas técnicas. Dado que es una 
característica más visible, que implica una acción concreta, esta pregunta fue la que tuvo una respuesta más relacionada con la planeación: “actualmente ya tiene una propuesta”, en concordancia con la escala con la que se contestaron las preguntas. Las medias, modas y desviaciones estándar de las nueve características de talento verde y de la variable "Talento Verde" se muestran en la tabla 6. De los resultados mostrados en dicha tabla, se puede concluir que los directivos de las empresas encuestadas cuentan con características de talento verde en algún grado. Con base en las respuestas, las empresas deben buscar capacitación para sus directivos acerca de las transformaciones químicas, físicas o biológicas en aire, agua y suelo en las que inciden los sistemas productivos de sus firmas y de esta manera encontrar formas de disminuir o eliminar los contaminantes emitidos al ambiente.

Tabla 6 Medias, modas y desviaciones estándar de las características de talento verde y de la variable "Talento Verde"

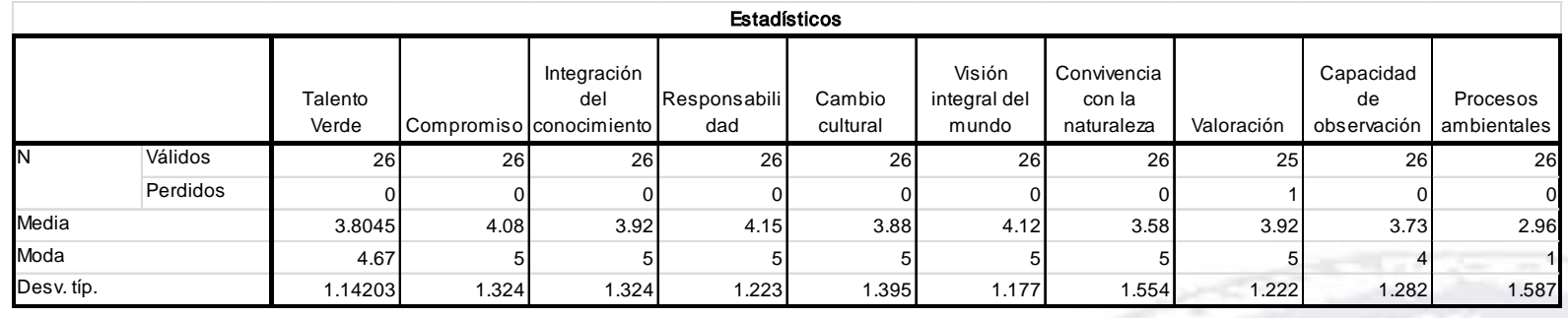

Fuente: Elaboración propia.

\section{Prácticas de cadena de suministro verde en las empresas encuestadas}

En cuanto a las prácticas de cadena de suministro verde (la variable se etiquetó como "Prácticas Verdes”), que desarrollan las empresas del oriente del estado de Morelos, por las respuestas dadas por los participantes y cuya media es 2.7038 (ver tabla 7), se puede afirmar que dichas prácticas solo están en un horizonte de planeación. Es decir, las respuestas emitidas se acercan, de acuerdo a su media aritmética y a la escala con que fueron medidas, a la respuesta “está siendo considerado en la actualidad”, lo que indica que las prácticas no se están llevando a cabo, pero si hay intención de llevarlas a cabo en algún momento.

La práctica que tiene la media más alta es la de eco diseño, con 3.1026, y es congruente con la media general, pues el diseño es más bien la planeación de un producto y por tanto la planeación de prácticas de sustentabilidad hacia el futuro. La siguiente media más alta, es la cooperación con los clientes, con 3.0385 que se relaciona con las preguntas sobre presiones institucionales, 
concretamente el mercado, que tienen una media de 3.5385. La administración ambiental interna es, como su nombre lo indica, la dirección al interior de las organizaciones y está, dada su media de 2.5753, en incipiente etapa de planeación. La práctica que obtuvo la media más baja, es la de compra verde, con 2.0192, lo que nos indica que las prácticas medio ambientales de los proveedores no se toman aun en cuenta al momento de la compra. Un resumen de las medias, modas y las desviaciones estándar de las prácticas de cadena de suministro verde se muestra en la tabla 7. En función de los resultados se recomienda primeramente, concretar las prácticas verdes con propuestas específicas, por ejemplo, trabajar en conjunto con sus proveedores para minimizar los impactos negativos al medio al medio ambiente; buscar la certificación ISO 14000 iniciando con la apertura de un departamento o un responsable de cuidado del medio ambiente. Para el diseño de nuevos productos se deberá considerar la disminución de su impacto ambiental midiendo la huella de carbono; fomentar una comunicación con los clientes para tomar en cuenta sus inquietudes con respecto al daño ecológico que provocan sus productos; y finalmente, considerar la venta del exceso de materia prima y de equipo obsoleto.

Tabla 7. Medias, modas y desviaciones estándar de las prácticas de cadena de suministro verde y de la variable "Practicas Verdes”

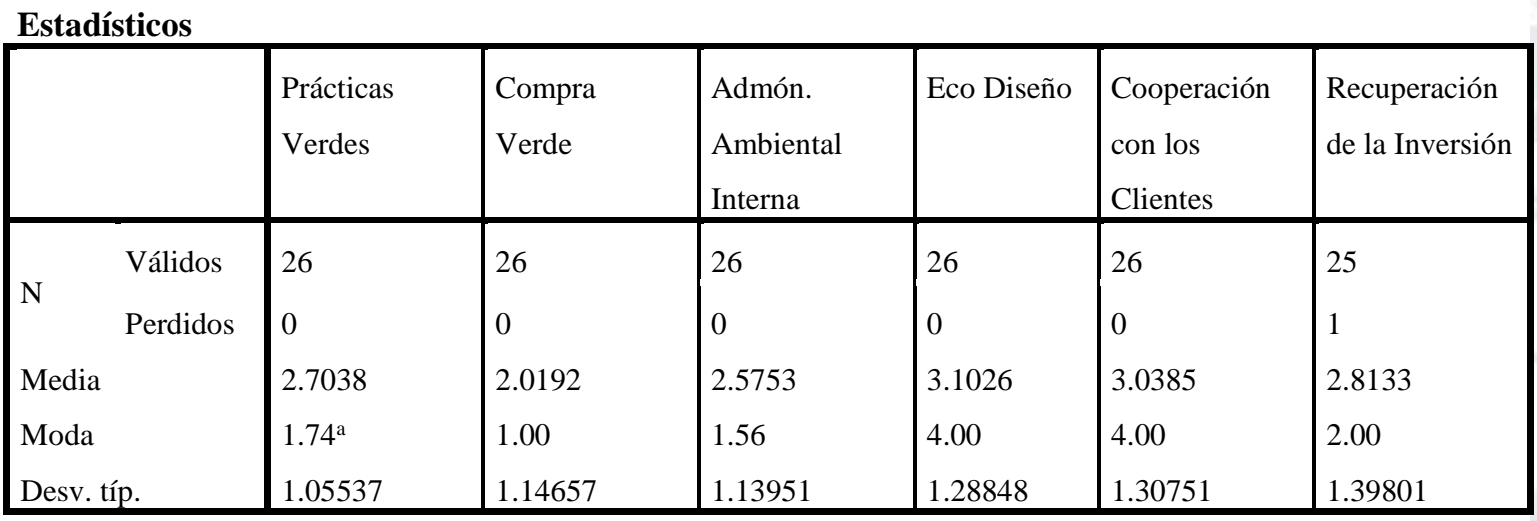

a. Existen varias modas. Se mostrará el menor de los valores.

Fuente: Elaboración propia.

\section{Presiones institucionales en las empresas encuestadas}

En cuanto a las presiones que reciben las empresas estudiadas, la media de esta variable reporta 3.4936 (ver tabla 8), lo que de acuerdo a la escala de respuesta, indica que las presiones son importantes para el trabajo de las compañías. La regulación, que comprende las leyes, es la presión que las empresas encuestadas consideran más importante, con una media de 3.5557 y la 
competidores es la que encuentran menos importante, con 3.4846 de media. El resumen estadístico de la encuesta, en cuanto a las presiones institucionales: mercado, regulación y competidores se muestra en la tabla 8. Algunas recomendaciones que emanan de estos datos es que las empresas encuestadas consideren la exportación de sus productos y la venta a clientes transnacionales así como las políticas medio ambientales de estos clientes. De la misma forma, reflexionar sobre las leyes de cuidado del medio ambiente en todos los niveles: internacionales, nacionales y locales. Finalmente, adherirse a los grupos industriales a los que pudieran pertenecer y verificar cuales son las políticas verdes que están promoviendo.

Tabla 8 Medias, modas y desviaciones estándar de la variable “Presiones Institucionales” y de los bloques que la componen

Estadísticos

\begin{tabular}{|ll|l|l|l|l|}
\hline & $\begin{array}{l}\text { Presiones } \\
\text { Institucionales }\end{array}$ & Mercado & Regulación & Competidores \\
\hline \multirow{2}{*}{ N $\quad$ Válidos } & 26 & 26 & 26 & 26 \\
Media & Perdidos & 0 & 0 & 0 & 0 \\
Moda & 3.4936 & 3.5385 & 3.5577 & 3.3846 \\
Desv. típ. & $2.00^{\mathrm{a}}$ & 5.00 & 4.00 & 4.00 \\
\hline
\end{tabular}

a. Existen varias modas. Se mostrará el menor de los valores.

Fuente: Elaboración propia.

En esta descripción de las categorías se encuentra que todas ellas tienen presencia en las empresas encuestadas. En un análisis más fino, en el apartado siguiente se muestran las pruebas de hipótesis para la aceptación o rechazo de las mismas.

\section{Pruebas de hipótesis}

Las hipótesis se probaron con estadística paramétrica, dado que los datos cumplen con los supuestos de normalidad para la variable dependiente y se trabajó con variables por intervalo (Hernández-Sampieri, Fernández-Collado y Baptista-Lucio 2010). La distribución de la variable dependiente "Prácticas Verdes" se comprobó con la prueba Shapiro Wilk para pequeñas muestras. La significancia que se obtuvo fue de 0.168 , que es mayor que 0.05 y por tanto indica que la variable se ajusta a una distribución normal. La práctica habitual asigna números enteros 
ordenados a los cuantificadores tipo Likert y los trata posteriormente como escala por intervalos (Cañadas-Osinki y Sánchez-Bruno 1998).

La H1 La presencia de características de talento verde en los directivos, se encuentra vinculada con la implementación de prácticas de cadena de suministro verde en empresas manufactureras con más de diez trabajadores de la zona oriente del estado de Morelos, México., presume una relación entre las variables que puede ser, aun mínimamente, causal. Las variables de esta hipótesis son: “Talento Verde” y “Prácticas Verdes”. Para probar esta hipótesis, se utilizó el coeficiente de correlación de Pearson. Los resultados se muestran en la tabla 9. Como se puede ver en dicha tabla, existe una correlación positiva considerable entre ambas variables ( $\mathrm{r}=0.699$, $\mathrm{N}=26, \mathrm{p}<0.01,2$ colas). Por todo lo anterior, la hipótesis 1 no se rechaza, es decir, existe una relación significativa entre los directivos con características de talento verde y la implementación de prácticas de cadena de suministro verde.

Tabla 9. Coeficiente de correlación de Pearson para las variables “Talento Verde” y “Prácticas Verdes”

\begin{tabular}{|ll|l|l|} 
Correlaciones & \multicolumn{2}{|l|}{} \\
& & $\begin{array}{l}\text { Prácticas } \\
\text { Verdes }\end{array}$ & Talento Verde \\
\hline & Correlación de Pearson & 1 & $.699^{* *}$ \\
Prácticas Verdes & Sig. (bilateral) & & $7.226 \mathrm{E}-005$ \\
& $\mathrm{~N}$ & 26 & 26 \\
& Correlación de Pearson & $.699^{* *}$ & 1 \\
Talento Verde & Sig. (bilateral) & $7.226 \mathrm{E}-005$ & \\
& $\mathrm{~N}$ & 26 & 26 \\
\hline
\end{tabular}

**. La correlación es significativa al nivel 0,01 (bilateral).

Fuente: Elaboración propia

La H2 Las presiones institucionales (mercado, legislación y competidores) están relacionadas de manera significativa con la implementación de prácticas de cadena de suministro verde en las empresas manufactureras con más de diez trabajadores de la zona oriente del estado de Morelos, México., hace referencia a una relación causa-efecto donde las presiones institucionales son causa de la implementación de prácticas de cadena de suministro verde. Para probar esta hipótesis, se utilizó el método de regresión lineal múltiple, donde la variable dependiente es 
"Prácticas Verdes” y las variables independientes son las presiones institucionales: "Mercado", "Regulación” y “Competidores”. En la tabla 10 se muestran los resultados del Análisis de Varianza / ANOVA con una significación de $6.792 \times 10^{-006}$, por lo que se puede concluir que existe una relación lineal entre la variable dependiente del modelo y las independientes. Los Análisis de Varianza / ANOVA para cada una de las variables: “Competidores”, "Mercado” y "Regulación”, se muestran en las tablas 11, 12 y 13; en todas, el nivel de significación es muy cercano a cero. Por todo lo anterior, la hipótesis 2 no se rechaza, es decir, la adopción de prácticas de cadena de suministro verde es un efecto de las presiones institucionales: mercado, regulación y competidores, en congruencia con los hallazgos de Zhu y Sarkis (2007).

Tabla 10. Análisis de varianza para prácticas de cadena de suministro verde y presiones institucionales

ANOVA $^{\text {a }}$
\begin{tabular}{|l|l|l|l|l|l|l|}
\hline Modelo & $\begin{array}{l}\text { Suma de } \\
\text { cuadrados }\end{array}$ & gl & $\begin{array}{l}\text { Media } \\
\text { cuadrática }\end{array}$ & F & Sig. \\
\hline \multirow{2}{*}{$\begin{array}{l}\text { Regresión } \\
1\end{array}$} & $\begin{array}{l}19.373 \\
\text { Residual } \\
\text { Total }\end{array}$ & $\begin{array}{l}8.472 \\
27.845\end{array}$ & 3 & 6.458 & 16.770 & $6.792{\mathrm{E}-006^{\mathrm{b}}}^{2}$ \\
\hline
\end{tabular}

a. Variable dependiente: Prácticas Verdes

b. Variables predictoras: (Constante), Competidores, Mercado, Regulación

Fuente: Elaboración propia

Tabla 11. Análisis de varianza para prácticas de cadena de suministro verde y competidores

\begin{tabular}{|c|c|c|c|c|c|c|}
\hline \multicolumn{7}{|c|}{ ANOVA $^{a}$} \\
\hline & & $\begin{array}{ll}\text { Suma de } & \text { de } \\
\text { cuadrados } & \\
\end{array}$ & gl & $\begin{array}{l}\text { Media } \\
\text { cuadrática }\end{array}$ & $F$ & Sig. \\
\hline \multirow{3}{*}{1} & Regresión & 16.715 & 1 & 16.715 & 36.040 & 3.380E-006 \\
\hline & Residual & 11.131 & 24 & .464 & & \\
\hline & Total & 27.845 & 25 & & & \\
\hline
\end{tabular}

a. Variable dependiente: Prácticas Verdes

b. Variables predictoras: (Constante), Competidores

Fuente: Elaboración propia 
Tabla 12. Análisis de varianza para prácticas de cadena de suministro verde y mercado

\begin{tabular}{|l|l|l|l|l|l|l|}
\hline \multicolumn{2}{|l|}{ ANOVA $^{\text {Modelo }}$} & $\begin{array}{l}\text { Suma de } \\
\text { cuadrados }\end{array}$ & gl & $\begin{array}{l}\text { Media } \\
\text { cuadrática }\end{array}$ & F & Sig. \\
\hline \multirow{4}{*}{$\begin{array}{ll}\text { Regresión } \\
\text { Residual }\end{array}$} & $\begin{array}{l}17.624 \\
10.221\end{array}$ & 1 & 17.624 & 41.384 & $1.187 \mathrm{E}-006^{\mathrm{b}}$ \\
& Total & 27.845 & 25 & .426 & & \\
\hline
\end{tabular}

a. Variable dependiente: Prácticas Verdes

b. Variables predictoras: (Constante), Mercado

Fuente: Elaboración propia

Tabla 13. Análisis de varianza para prácticas de cadena de suministro verde y regulación

\begin{tabular}{|c|c|c|c|c|c|c|}
\hline \multicolumn{7}{|c|}{ ANOVA $^{a}$} \\
\hline & & \begin{tabular}{|ll} 
Suma & de \\
cuadrados & \\
\end{tabular} & gl & $\begin{array}{l}\text { Media } \\
\text { cuadrática }\end{array}$ & $F$ & Sig. \\
\hline \multirow{3}{*}{1} & Regresión & 17.049 & 1 & \multirow{3}{*}{$\begin{array}{l}17.049 \\
.450\end{array}$} & \multirow[t]{3}{*}{37.897} & \multirow[t]{3}{*}{$2.324 \mathrm{E}-006^{\mathrm{b}}$} \\
\hline & Residual & 10.797 & 24 & & & \\
\hline & Total & 27.845 & 25 & & & \\
\hline
\end{tabular}

a. Variable dependiente: Prácticas Verdes

b. Variables predictoras: (Constante), Regulación

Fuente: Elaboración propia

La H3 Las presiones institucionales y la presencia de directivos con talento verde tienen una influencia significativa en la implementación de prácticas de cadena de suministro verde en las empresas manufactureras con más de diez trabajadores en la zona oriente del estado de Morelos, México., hace referencia a una combinación, tanto del talento verde como de las presiones institucionales, para la implementación de prácticas de cadena de suministro verde. Por lo que $\mathrm{H} 1$ es un subconjunto de H3. La variable dependiente es "Prácticas Verdes" y las independientes son "Presiones Institucionales” y “Talento Verde”. Se utilizó nuevamente la regresión lineal múltiple. La tabla 14 muestra el Análisis de Varianza / ANOVA del modelo y dado que la significación es de $2.845 \times 10^{-007}$, existe una relación lineal entre la variable dependiente y el conjunto de independientes. Los Análisis de Varianza / ANOVA para cada una de las variables: "Presiones Institucionales”, y “Talento Verde”, se muestran en las tablas 15 y 16; en todas, el nivel de significación es muy cercano a cero. Por todo lo anterior, la hipótesis 3 
no se rechaza, es decir, la combinación de talento verde y presiones institucionales, son causa de la implementación de prácticas de cadena de suministro verde.

Tabla 14. Análisis de varianza para prácticas de cadena de suministro verde, presiones institucionales y talento verde.

ANOVA $^{\mathrm{a}}$

\begin{tabular}{|ll|l|l|l|l|l|}
\hline \multicolumn{2}{|l|}{ Modelo de gl } & $\begin{array}{l}\text { Suma } \\
\text { cuadrados }\end{array}$ & $\begin{array}{l}\text { Media } \\
\text { cuadrática }\end{array}$ & F & Sig. \\
\hline \multirow{2}{*}{1} & Regresión & 20.337 & 2 & 10.168 & 31.148 & $2.845 \mathrm{E}-007^{\mathrm{b}}$ \\
& Residual & 7.508 & 23 & .326 & & \\
& Total & 27.845 & 25 & & & \\
\hline
\end{tabular}

a. Variable dependiente: Prácticas Verdes

b. Variables predictoras: (Constante), Talento Verde, Presiones Institucionales Fuente: Elaboración propia.

Tabla 15. Análisis de varianza para prácticas de cadena de suministro verde y presiones institucionales

ANOVA $^{\mathrm{a}}$

\begin{tabular}{|ll|l|l|l|l|l|}
\hline Modelo & $\begin{array}{l}\text { Suma de } \\
\text { cuadrados }\end{array}$ & gl & $\begin{array}{l}\text { Media } \\
\text { cuadrática }\end{array}$ & F & Sig. \\
\hline \multirow{2}{*}{1} & Regresión & 19.011 & 1 & 19.011 & 51.650 & $1.993 \mathrm{E}-007^{\mathrm{b}}$ \\
& Residual & 8.834 & 24 & .368 & & \\
& Total & 27.845 & 25 & & & \\
\hline
\end{tabular}

a. Variable dependiente: Prácticas Verdes

b. Variables predictoras: (Constante), Presiones Institucionales

Fuente: Elaboración propia.

Tabla 16. Análisis de varianza para prácticas de cadena de suministro verde y talento verde ANOVA $^{\mathrm{a}}$

\begin{tabular}{|ll|l|l|l|l|l|}
\hline Modelo & $\begin{array}{l}\text { Suma de } \\
\text { cuadrados }\end{array}$ & gl & $\begin{array}{l}\text { Media } \\
\text { cuadrática }\end{array}$ & F & Sig. \\
\hline \multirow{4}{*}{1} & Regresión & 13.587 & 1 & 13.587 & 22.869 & $7.226 \mathrm{E}-005^{\mathrm{b}}$ \\
& Residual & 14.259 & 24 & .594 & & \\
& Total & 27.845 & 25 & & & \\
\hline
\end{tabular}

a. Variable dependiente: Prácticas Verdes

b. Variables predictoras: (Constante), Talento Verde

Fuente: Elaboración propia.

\section{Conclusiones}

El cuidado del medio ambiente es una actividad que engloba a todas las áreas del conocimiento.

En este trabajo se pudieron conjuntar metodologías cuantitativas y cualitativas para obtener resultados sobre el factor humano responsable de la implementación de prácticas de cadena de suministro verde y de las prácticas que en sí realizan las empresas, además de encontrar la relación entre las variables asociadas a ello. 
Para la zona oriente del estado de Morelos, México, las prácticas de cadena de suministro verde se encuentran aún en etapa de planeación; los directivos de las empresas encuestadas presentan características de talento verde en acciones que llevan a cabo en algún grado. La característica con mayor grado de incidencia, es la responsabilidad, en tanto que la característica que se detecta como de menor incidencia en los directivos de las empresas de la zona oriente del estado de Morelos es la capacitación sobre transformaciones físicas, químicas o biológicas en el aire, agua o suelo que se producen en las empresas en las que laboran.

El “Talento Verde” y las “Prácticas Verdes” son variables que se relacionan, es decir, existe una relación estadísticamente positiva significativa entre ambas variables. Estos resultados permiten no rechazar la hipótesis 1: La presencia de características de talento verde en los directivos, se encuentra vinculada con la implementación de prácticas de cadena de suministro verde en empresas manufactureras con más de diez trabajadores de la zona oriente del estado de Morelos, México.

Las presiones institucionales: mercado, regulación y competidores, son causa de la implementación de prácticas de cadena de suministro verde, de acuerdo al método de regresión lineal múltiple. Esta afirmación dada por los resultados estadísticos, permite no rechazar la hipótesis 2: Las presiones institucionales (mercado, legislación y competidores) están relacionadas de manera significativa con la implementación de prácticas de cadena de suministro verde en las empresas manufactureras con más de diez trabajadores de la zona oriente del estado de Morelos, México.

Los análisis estadísticos aplicados a las variables “Talento Verde”, "Presiones Institucionales” y “Prácticas Verdes”, reconocen una relación causal que permite no rechazar la hipótesis 3: Las presiones institucionales y la presencia de directivos con talento verde tienen una influencia significativa en la implementación de prácticas de cadena de suministro verde en las empresas manufactureras con más de diez trabajadores en la zona oriente del estado de Morelos, México. Por lo tanto puede afirmarse que: en las empresas manufactureras de más de diez trabajadores en la zona oriente del estado de Morelos, México, la presencia de características de talento verde en los directivos, se encuentra vinculada con la implementación de prácticas de cadena de suministro verde; asimismo las presiones institucionales (mercado, legislación y competidores) están relacionadas de manera significativa con la implementación de prácticas de cadena de 
suministro verde, por lo que ambas variables tienen una influencia significativa en la implementación de las mencionadas prácticas.

Estas conclusiones, si bien se encuentran acotadas a un tiempo y un espacio, puesto que se presenta un estudio de caso, se observa la posibilidad de que en futuras investigaciones se incluya la aplicación de la encuesta en todo el estado de Morelos, México y en todo el país, para conocer el estado que guardan las prácticas verdes en las cadenas de suministro asentadas en territorio mexicano. Además puede valorarse la realización de un trabajo operativo para la adopción de prácticas verdes en las pequeñas y medianas empresas que muestran interés por el cuidado del medio ambiente dentro del estado de Morelos, México. Incluso, sería conveniente enriquecer la encuesta considerando bloques sobre transporte, emisiones de gases de efecto invernadero, tratamiento de agua y contaminación de suelos.

\section{Referencias}

Ahi, Payman y Cory Searcy. (2013). A comparative literature analysis of definitions for green and sustainable supply management. Journal of Cleaner Production 52: 329-341

Barney, Jay. (1991). Firm resources and sustained competitive advantage. Journal of Management 17 (1): 99-120.

Beamon, Benita M. (1999). Designing the green supply chain. Logistics Information Management 12(4): 332-342. http://doi.org/10.1108/09576059910284159

Ben Brik, Anis; Kamel Mellah y Belaid RettabMellah. (2013). Drivers of Green Supply Chain in Emerging Economies. Thunderbird International Business Review 55(2): 123-136

Cañadas-Osinski, Isabel y Alfonso Sánchez-Bruno. (1998). Categorías de respuestas en escalas tipo Likert. Psicothema 10(3): 623-631.

Centro de Estudios de las Finanzas Públicas. (2015). Impuestos ambientales en México y el mundo. México: LXIII Legislatura Cámara de Diputados.

Chopra, Sunil y Peter Meindl. (2008). Administración de la cadena de suministro. México: Pearson Prentice Hall.

Christmann, Petra. (2000). Effects of 'best practices' of environmental management on cost competitiveness: the role of complementary assets. Academy of Management Journal 43: 663880. 
CONACYT. (2015). Empresas. http://www.conacyt.mx/index.php/el-conacyt/desarrollotecnologico-e-innovacion (2 de noviembre).

Daily, Bonnie F. y Su-chun Huang. (2001). Achieving sustainability through attention to human resource factors in environmental management. International Journal of Operations \& Production Management 21(12): 1539-1552. http://doi.org/10.1108/01443570110410892

Delmas, Magali y Arturo Keller. (2005). Free riding in voluntary environmental programs: the case of the US EPA WasteWise program. Policy Sciences 38: 91-106.

DiMaggio, Paul J. y Walter W. Powell. (1983). The Iron Cage Revisited: Institutional Isomorphism and Collective Rationality in Organizational Fields. American Sociological Review 48: $147-160$.

DOF (Diario Oficial de la Federación de México) (2009). Acuerdo por las que se establece la estratificación de las micro, pequeñas y medianas empresas. Junio 30. http://www.dof.gob.mx/nota_detalle.php?codigo=5096849\&fecha=30/06/2009.

El Saadany, A.M.A., Jaber, M.Y. y Booney, M. (2011). Management Research Review 34(11): 1202-1221. http://dx.doi.org/10.1108/01409171111178756

Emerson, Richard M. (1962). Power-Dependence Relations. American Sociological Review 27(1): 31-41.

Gattorna, John. (2006). Supply chains are the business. Supply Chain Management Review 6(10): 42-49.

Gobierno del Estado de Morelos. (2013). Fideicomiso Ejecutivo del Fondo de Competitividad y Promoción del Empleo. Acta de la Quinta Sesión Ordinaria del 2013. http://www.transparenciamorelos.mx/sites/default/files/Ejecutivo_Auxiliar/FIDECOMP/oe3/AC TA\%205A\%200RD\%2013\%20CT\%20FIDECOMP_0.pdf

Hair, Joseph F., Rolph E. Anderson, Ronald L.Tatham y William Black. (1992). Multivariate Data Analysis with Readings. New York: Macmillan Publishing Company.

Handfield, Robert B., Steve V. Walton, Lisa K. Seegers y Steven A. Melnyk. (1997). Green value chain practices in the furniture industry. Journal of Operations Management 15 (4): 293-315. doi:10.1016/S0272-6963(97)00004-1 
Hernández-Sampieri, Roberto; Carlos Fernández-Collado y Pilar Baptista-Lucio. (2010). Metodología de la investigación. México, D.F. : McGraw Hill.

Hervani, Aref A., Marilyn M. Helms y Joseph Sarkis. (2005). Performance measurement for green supply chain management. Benchmarking: An International Journal 12 (4): 330-353. http://dx.doi.org/10.1108/14635770510609015

Hoek, Remko I. Van. (1999). From reversed logistics to green supply chains. Supply Chain Management: An International Journal 4(3): 129-135. http://doi.org/10.1108/13598549910279576

INEGI (Instituto Nacional de Estadística y Geografía). (2014). Directorio Estadístico Nacional de Unidades Económicas. http://www3.inegi.org.mx/sistemas/mapa/denue/default.aspx (9 de febrero de 2015)

Kotzab, Herbert. (2005). The role and importance of survey research in the field of supply chain management. En Research methodologies in supply chain management (125-137). Germany: Physica-Verlang Heidelberg.

Kumar, Sameer; Steve Teichman y Tobias Timpernagel. (2012). A green supply chain is a requirement for profitability. International Journal of Production Research 50(5): 1278-1296. http://doi.org/10.1080/00207543.2011.571924

Lakhal, Salem Y., Souad H'Mida y M. Rafiqul Islam. (2007). Green supply chain parameters for a Canadian petroleum refinery company. International Journal of Environmental Technology and Management 7(1-2): 56-67.

Laosirihongthong, Tritos; Dotun Adebanjo y Keah Choon Tan. (2013). Green supply chain management practices and performance. Industrial Management \& Data Systems 113 (8): 10881109.

Lee, Su-Yol. (2008). Drivers for the participation of small and medium-sized suppliers in green supply chain initiatives. Supply Chain Management: A International Journal 13(3): 185-198. DOI: $10.1108 / 13598540810871235$

Melnyk, Steven A., Robert P. Sroufe y Roger Calantone. (2003). Assessing the impact of environmental management systems on corporate and environmental performance. Journal of Operations Management 21: 329-351.

Murphy, Paul R. Jr. y A. Michel Knemeyer. (2015). Logística contemporánea. México: Pearson Educación. 
Pagell, Mark y David Gobeli. (2009). How plant managers' experiences and attitudes toward sustainability relate to operational performance. Production and Operations Management 18(3): 278-299. http://doi.org/10.1111/j.1937-5956.2009.01050.x

Pérez-López, Cesar. (2008). Técnicas de análisis multivariante de datos: aplicaciones con SPSS. Madrid: Pearson Educación. S.A.

Sarkis, Joseph; Pilar Gonzalez-Torre y Belarmino Adenso-Diaz. (2010). Stakeholder pressure and the adoption of environmental practices: The mediating effect of training. Journal of Operations Management 28(2): 163-176. http://doi.org/10.1016/j.jom.2009.10.001

SEMARNAT. (2013). Estrategia Nacional de Cambio Climático. México: Autor.

Srivastava, Samir K. (2007). Green Supply Chain Management: A State of the Art Literature Review. International Journal of Management Reviews 9(1): 53-80. DOI: 10.1111/j.14682370.2007.00202.x

Tapia-Uribe, Medardo. (2006). Morelos capital del conocimiento. Cuernavaca: CRIM-UNAM.

Torres-Salazar, Maria del Carmen; Ana Esther Escalante-Ferrer; Elías Olivares-Benítez y Juan Carlos Pérez-García. (2015). Talento verde: caracterización y búsqueda. Revista Electrónica Nova Scientia 14 (7): 694-709.

Zhu, Qinghua y Joseph Sarkis. (2007). The moderating effects of institutional pressures on emergent green supply chain practices and performance. International Journal of Production Research 45(18-19): 4333-4355. DOI: 10.1080/00207540701440345

Zhu, Qinghua y Yong Geng. (2001) Integrating environmental issues into supplier selection and management: a study of Large and Medium-sized State-owned Manufacturers in China. Greener Management International 9: 27-40. 